\title{
Congenital luxation of the knee: with regard to a case.
}

Elvis Rafael Ávila Santana1, Eduardo Albisu Santana², Luis Felipe Barranco ${ }^{3}$, Norisley Céspedes Carbalido ${ }^{4}$

${ }^{1}$ First Degree Specialist in Orthopedics and Traumatology. Instructor teacher. William Soler Pediatric Hospital.

${ }^{2}$ II Degree Specialist in Orthopedics and Traumatology. Assistant Professor. William Soler Pediatric Hospital.

${ }^{3}$ II Degree Specialist in Orthopedics and Traumatology. Assistant teacher. William Soler Pediatric Hospital.

First Degree Specialist in Orthopedics and Traumatology. William Soler Pediatric Hospital.Havana, Cuba 2020

*Corresponding Author: Elvis Rafael Ávila Santana, 1First Degree Specialist in Orthopedics and Traumatology. Instructor teacher. William Soler Pediatric Hospital. Havana, Cuba 2020.

Received date: July 07, 2020; Accepted date: August 07, 2020; published date: August 11, 2020

Citation: E R Á Santana, E A Santana, L F Barranco, N C Carbalido.(2020). Congenital luxation of the knee: with regard to a case. Clinical Orthopaedics and Trauma Care. 2(2); DOI:10.31579/2694-0248/007

Copyright: @2020 Elvis Rafael Ávila Santana, This is an open-access article distributed under the terms of the Creative Commons Attribution License, which permits unrestricted use, distribution, and reproduction in any medium, provided the original author and source are credited.

\section{Abstract}

The congenital luxation of the knee is an unfrequent affection, existing many theories about it's appearance. In some cases, it is considered an isolated deformation although it is relativly frequent to associate it to other congenital malformations, such as the dysplasia on the development of the hip, feet deformities or dislocation of the elbow. The treatment of this kind of affection depends on the seriousness and flexibility of the fondness, having like therapeutic lines the placing of serial casts, by using Pavlik's armor or plastia of the four headed muscle on severe cases. We present a case of congenital luxation of the knee treated by the use of serial casts, with a favorable evolution.

Key words: congenital luxation of the knee; congenital deformity of the knee; treatment; congenital knee dislocation; congenital knee deformity;

\section{Introduction}

Congenital knee dislocation is a rare disease, there are several theories that try to explain the possible causes of the appearance of this deformity such as: heredity, abnormal position of the fetus during pregnancy, bone configuration of the fetus' knee and less likely knee injury during childbirth. [1,2]

The most severe cases are believed to be related to mesenchymal alterations during fetal development, as they are frequently associated with other deformities or syndromes. The milder ones, on the other hand, could be due to abnormal intrauterine positions, so other concomitant diseases related to these, such as developmental dysplasia of the hip, should be ruled out. [3,4]

Treatment is started as soon as possible, preferably from birth through manipulations, serial casting, Pavlik harness and in severe cases quadriceps femoris plasties. [2, 5,6]

We present a case of congenital knee dislocation treated with serial casts placed from the nursery in the neonatology service, progressing favorably.

\section{Case}

We are called from the neonatology service of the Enrique Cabrera Cossío Clinical Surgical Hospital to evaluate the newborn for presenting deformity at the level of the left knee.

23-year-old mother, with health history, normal pregnancy, nulliparous, normal ultrasound studies, breech presentation. Cesarean delivery (arrested descent of presentation) at 40.1 weeks, patient weight at birth 3270 grams, length $49 \mathrm{~cm}$, head circumference $36 \mathrm{~cm}$, OFF $9 / 9$.

Physical examination: Hyperextension of the left knee, passive flexion of the same to approximately 700 (Figure 1), left calcaneal-valgus foot, no alterations in the right lower limb, negative examination of the hips. 


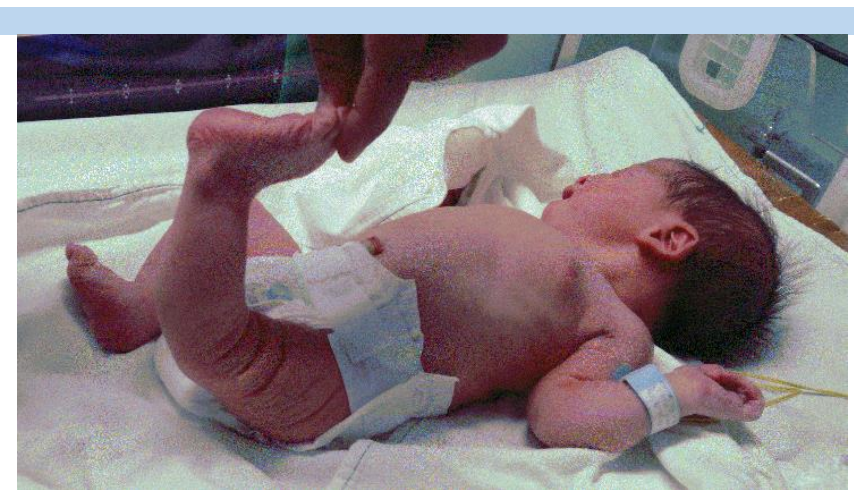

Imaging Studies: Plain X-rays are performed anteroposterior and lateral view of the right knee and bony pelvis (Figures 2 and 3).
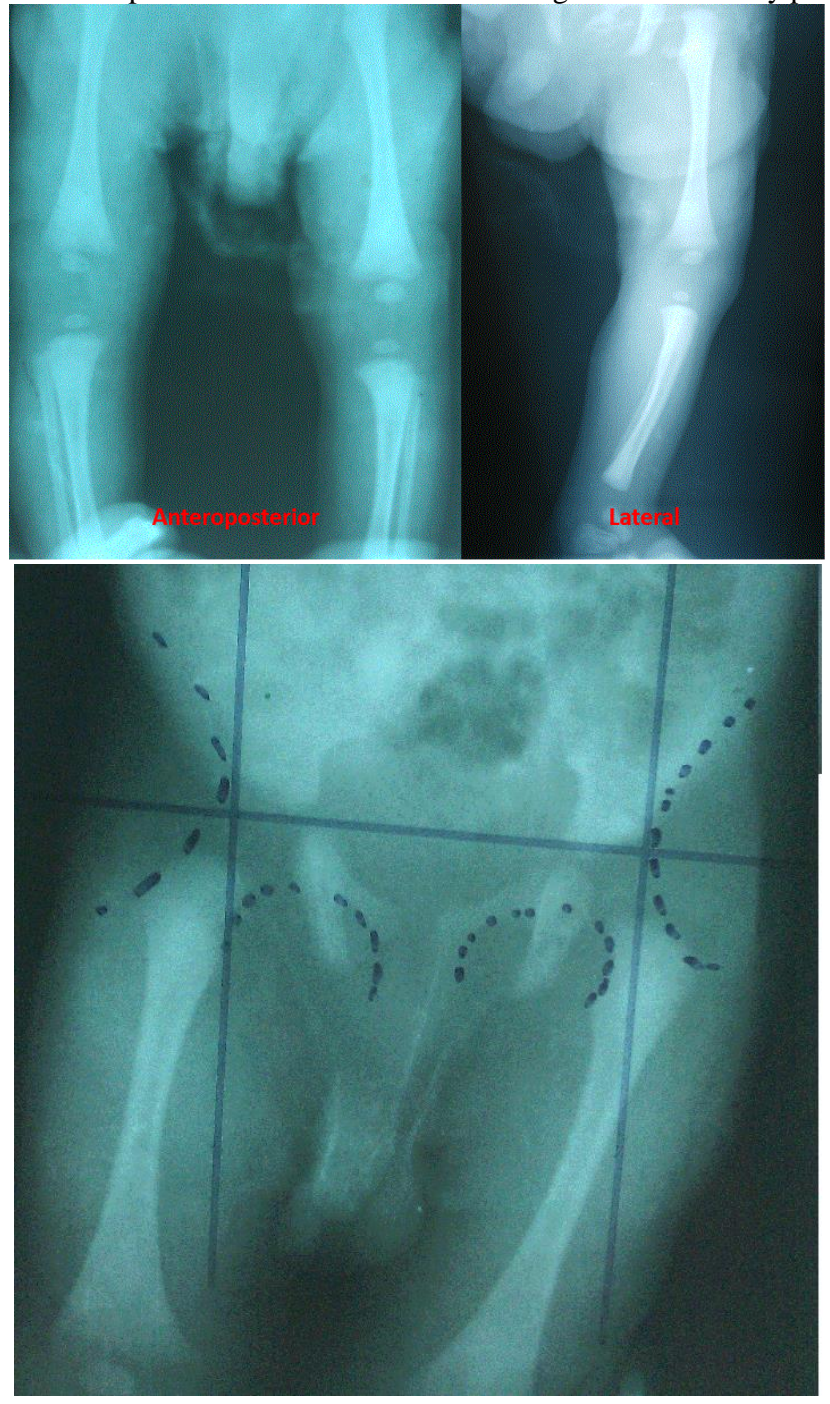

Leveuf's type A congenital knee dislocation was found (Figure 4)

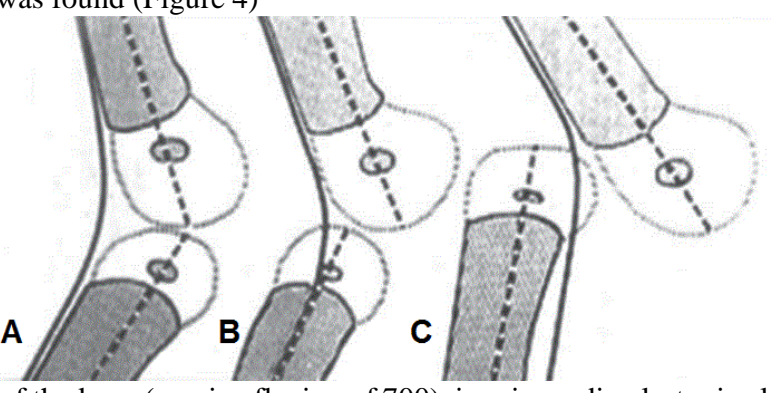

Conduct to follow: Given the easy reduction of the knee (passive flexion of 700), inguinopedic plaster is placed (Figure 5), it is changed fortnightly for 6 weeks achieving a passive knee flexion greater than 900 (Figure 6) Therefore, it was decided to withdraw immobilization and indicate rehabilitation 
of the affected knee, not only achieving a good reduction of the congenital knee dislocation but also of the calcaneus-valgus. Periodic follow-ups were carried out until autonomous ambulation, obtaining a knee with good stability, functionality and without delay in the patient's psychomotor development.
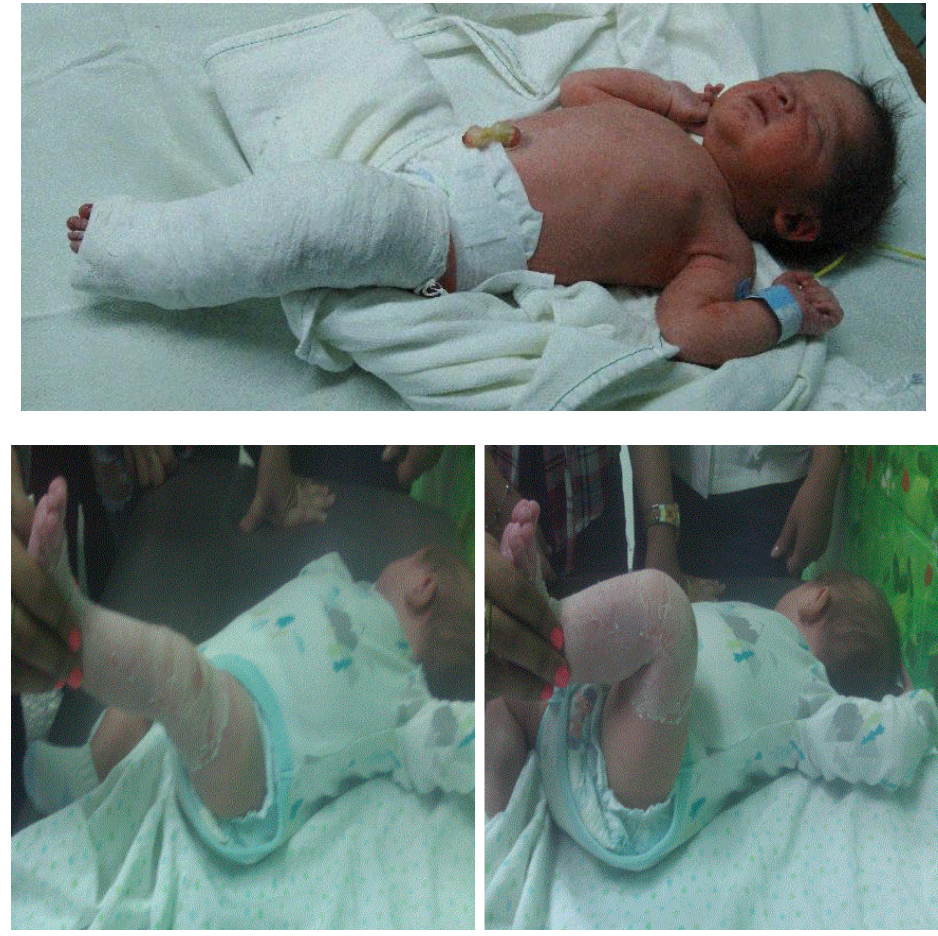

\section{Discussion}

Congenital knee dislocation is a rare condition where there is an antero-external translation of the tibia, presenting an incidence of one case per 100,000 live births, the causes that could favor the development of said deformity are still an enigma. There are several theories that could explain the appearance of this disease from the bad intrauterine position (such as the position of the buttocks) to defects in mesenchymal development. In the case of the patient in question, I present an inadequate intrauterine position (breech presentation) which, according to the literature, may be one of the possible favorable causes of said deformity. [3, 7, 8,9]

A thorough physical examination is necessary since this deformity can be associated with other diseases such as: developmental dysplasia of the hip (45\%), breech deformities (31\%), and congenital elbow dislocation (10\%). In severe cases we can find the association of entities such as arthrogryposis, Down syndrome, Larsen syndrome.

Congenital knee dislocation can be bilateral, which in most cases is due to the absence of the cruciate ligaments in hypermobility syndromes, but unilateral is more frequent, it is usually more stable once the reduction has been performed. [8.10]

The diagnosis of congenital knee dislocation is generally not difficult in most cases, it is enough with the inspection where the hyperextension of the knee is verified, which clearly differs from the normal position of the newborn, it should be seeing the degree of passive flexion of the same which helps us together with the imaging studies to classify said deformity (Leveuf classification (Figure2).Type A is the most common and is not really a dislocation but a congenital hyperextension with passive flexion limited to 45-900, as in our case. In type B there is a congenital subluxation with loss of joint congruence and in type $C$ there is a complete loss of it. [5, 6,11]

Regarding the treatment, it will depend on the degree of severity of the deformity. The degree of quadriceps contracture will determine the possibility of reduction by applying gentle longitudinal traction in addition to anteroposterior pressure on the tibia until the knee is flexed. In the event that the dislocation is irreducible, the application of soft or skeletal traction prior to immobilization with a plaster has been described. On the other hand, if it is a subluxation or reducible dislocation, it can be immobilized with an inguinopedic cast until passive flexion accepted by the patient at the time of reduction, changing them seriously until a 900 flexion of the knee was achieved. Said treatment was indicated for the patient in question, obtaining satisfactory results. There are authors who prefer the use of the Pavlik harness in Leveuf type A and B, with which they have obtained good results. $[10,11]$ Open reduction would be indicated if $90 \mathrm{o}$ of flexion is not obtained after serial manipulations until the fourth week. There are several techniques described such as the V en Y plasty (VYC) of the quadriceps tendon associated with an arthrotomy to free the ligamentous structures displaced anteriorly, while others advocate femoral shortening in order to avoid complications derived from quadriceps scarring. . Regarding the different quadriceps plasty techniques, percutaneous quadriceps recession (CPR) carries less morbidity (such as adhesions and blood loss) compared to VYC, while VYC is more successful in obtaining and maintaining the reduction in severe and resistant cases. [6, 7,12] A clear association has not been found between the severity of the degree of joint discontinuity at the time of diagnosis and the surgical indications, since there are studies where a type $\mathrm{C}$ dislocation evolved satisfactorily with treatment with casts. Abdelalaziz and Samir described a new classification according to the degrees of passive flexion that can be obtained at the time of diagnosis: grade I if $>900$, grade II if 30-900 and grade III if $<300$. This classification system should also function as an indication Therapeutic: GI would be subsidiary to treatment with serial casts, GII in neonates up to one month of age, initially treated with casts and mobility is reassessed after four manipulations: if $>90 \mathrm{o}$, conservative treatment is maintained, while $\mathrm{CPR}$ is recommended if it is $<90 \mathrm{o}$. In GIII and relapses, they refer that a VYC should be performed. [8, 10, 11,12]

\section{References}


1. Rivas-Laso JA, López-Bargiela P, Rodríguez-Rodríguez ML, Castro-Torre M, González-Herranz P. (2009) Congenital knee dislocation: review. Ortop Gallega Act. 5 (2): 49-51.

2. Elmada ğ M, Ceylan HH, Erdil M, İmren Y, Bilsel K, Tuncay I. (2013) Congenital dislocation of knee. Eur J Gen Med. 10 (3): $164-166$.

3. Biswas D, Akter FA, Roy A, Aolad FR, Khan SA. (2014) Congenital hyper-extension with subluxation of the knees - report of 2 cases. Mymensingh Med J.; 23 (4): 811-813.

4. Cheng CC, Ko JY. (2010) Early reduction for congenital dislocation of the knee within twenty-four hours of birth. Chang Gung Med J. 33 (3): 266-273.

5. Wilson PL, Rathjen KE. (2014) Disorders of the knee. In: Herring JA, editor: Tachdjian's pediatric orthopedics. 5th ed. Volume I. Texas: Saunders. 682-686.

6. Abdelaziz TH, Samir S. (2011) Congenital dislocation of the knee: a protocol for management based on degree of knee flexion. J Child Orthop. 5 (2): 143-149.

7. Tercier S, Shah H, Joseph B. (2012) Quadricepsplasty for congenital dislocation of the knee and congenital quadriceps contracture. J Child Orthop. 6 (5): 397-410.

8. Sud A, Chaudhry A, Mehtani A, Tiwari A, Sharma D. (2009) Functional outcome following quadriceps tendon lengthening in congenital dislocation of the knee, with special reference to extensor weakness. Strategies Trauma Limb Reconstr. 4 (3): 123-127.

9. Sudesh P, Singh D, Goni V, Rangdal S, Chaudhary S. (2013) Late presentation of congenital dislocation of the knee: a case report. J Knee Surg. 26 Suppl 1: S1-5.

10. Sud A, Kumar N, Mehtani A. (2013) Femoral shortening in the congenital dislocation of the knee joint: results of mid-term follow-up. J Pediatr Orthop B. 22 (5): 440-444.

11. Patwardhan S, Shah K, Shyam A, Sancheti P. (2015) Assessment of clinical outcome of percutaneous needle quadriceps tenotomy in the treatment of congenital knee dislocation. Int Orthop. 39 (8): 1587-1592.

12. Kazemi SM, Abbasian MR, Hosseinzadeh HR, Zanganeh RF, Eajazi A, Daftari-Besheli L. (2010) Congenital dislocation of the knee in a 16year old girl. Orthopedics. 33 (5). 